\title{
FACTORS AFFECTING THE PERCEPTION OF PHOTOGENIC QUALITY IN PUBLIC SPACES; Insights from Arcade Independence Square, Colombo
}

\author{
DE SILVA. G.G.S ${ }^{1}$ \& HETTIARACHCHI. A.A ${ }^{2}$
}

1,2 Department of Architecture, University of Moratuwa, Sri Lanka 1gshanaka2@live.com, 2anishkah@uom.lk

\begin{abstract}
Photography having one less dimension than Architecture, is one of the main elements of modern-day public user. With the growth of social media, the influence of media on the daily tasks of the average user has increased exponentially in the last decade. This study analysed the perception of public spaces in this new context of photography and social media, in relation to the photogenic quality of a space. The identification of reasons behind the perception of photogenic quality in public places would help both the designers and the government administration to create better public spaces. The study was executed with reference to three selected spaces of the Arcade Independence Square in Colombo considering 3 user groups ( $n=94$ ) selected based on their knowledge base namely; architecture, photography and a neutral group from general public adopting an online questionnaire survey. The findings identified visual elements of the space/composition as the significant reason behind the perception of the photogenic quality of a space followed by cultural influence and social media.
\end{abstract}

Keywords: "Visual Perception." "Public Spaces." "Photography." "Situationism." "Social Media."

\section{Background}

Modern architecture makes up the essence of the living cities in the world and these are crafted by architects to be in line with the living standards of the occupants. This process of crafting comprises of functional workability married with the aesthetic beauty of the spaces. The ultimatum gives the iconic architectural monuments in modern cities. Not only they pop out from their physical context to give definition to the built environment but also, singlehandedly create an identity of impression of the entire city. Regardless of their three-dimensional spatial sense, the image created in the mind of tourists is a twodimensional frame. This frame augments the reality to create the perfect eye-catching 'destination image'.

The study of destination image creation has been widely examined in the field of tourism due to its importance in the process of decision making for potential visitors when choosing a destination, and tourists' subsequent evaluation and restructuring of the destination image after their experience at a destination (Godfrey \& Gretzel, 2010). This was interpreted in the 'society of the spectacle', the work by Guy Debord as the concept of a 'spectacle'. The idea of situationism is discussed by the author to contradict the perception of reality. "All that once directly lived has become mere representation" (Debord, 1967). The idea of spectacle is not a result of a series of images, but it is a connection among people which was mediated via images. Accordingly, Debord observed that the images influence human lives, relationships and beliefs on a daily basis. This very basic idea of a spectacular society gives the essence for the architects knowingly or unknowingly to create the spectacle that the society needs by the image of manipulated spaces. A moment is a sense of self consciousness of existence within a particular environment or ambiance (Debord, 1967).

The modern 'society of spectacle' being envisioned as the global village, is nourished by the internet. People navigate the cities and public spaces from Google Maps based on the ratings of previous 
users and the images that provide a sense of notion of that place. This process of place discovery occurs with the visual sensitivity of our optical senses to recognize the architectural elements and the public space that defines the spectacle. This visual sense is based on the photographic representation of previous users, which are most of the time the general public, as opposed to architectural photographers who know how to capture a proper frame and the highlights from the essence of space. But the handful of architectural photographs are a mere source of marketing on marketing and advertising platforms that are supposed to deliver the elements of the spectacle into the society but fails in close proximity due to the lack of engagement with the modern social life of the spectacle users. Therefore, the users perceive the notion of the space from the collective effort of nonprofessional photographs which are mere moments of selfsatisfaction of a particular user.

\subsection{RESEARCH NEED}

Architectural sense can be individually adapted and defined according to the user's personal experiences through their course of life. The attention of the architect to create a 'symbol' within each space is exploited by the user perception to create an artefact of their own emotional attachment. Different public spaces in Colombo create different spectacles. The perceiving user groups of these public spaces vary in categories due to the spatial gentrification of public spaces in Colombo. The gentrified public spaces are accompanied by the middle class to richer social groups while the less gentrified spaces have a diversity of human interactions and social groups.

Transformation from global village to a global family with a single touch from a device has revolutionised the way human minds work. The application of new concepts into life has eluded society into an alienization of their physical surroundings along with the life that contained in that envelop of surrounding.

Virtual relationships juxtaposed the notion of physical relationships. The alienation of the spectator, which reinforces the contemplated objects that result from his own unconscious activity, works in such a way that: the more he contemplates, the less he lives; the more he identifies with the dominant images of need, the less he understands his own life and his own desires (Debord, 1967). The social media thus re-unites the separated, but it reunites them only in their separateness. The interactions people are making via social media have transformed the way the people interact in real life. Usual spatial practices are being rearranged to meet the needs of social media. Parochial spaces are territories characterized by 'a sense of commonality among acquaintances and neighbours who are involved in interpersonal net-works that are located within communities' (Humphreys, 2010).

As expressed by Selay, (Tok, Kaplan, \& Taneli, 2010) architecture is primarily concerned with photography as a tool for optical realist recording as a means of documenting the immediate material environment. Photography helps in the design process for architects and also is a key factor in the commercial marketing of architectural designs. In his research Selay (Tok, Kaplan, \& Taneli, 2010) identified that the process of reading an image depends and varies from person to person. Reading of a photograph is a process of interpretation and elicitation. It is an intentional effort to comprehend the sensory experience represented in the photo coupled with an awareness and accounting of the feelings, thoughts, memories and emotions which are stimulated through engaging with that experience. Thus, more the knowledge a person has on the cultural, political, economic, social backgrounds of a pictorial depiction, the image readability tends to become a projection of this awareness. Inevitably, we bring our own personal and cultural baggage into our interpretation of photographs and shared understandings of shared places cannot be taken for granted (Tok, Kaplan, \& Taneli, 2010). Therefore, photography can also be termed as a process of sharing perspectives. Similar to all the elements of a sketch being intentional for the artist, a photograph also has intentional elements, when taken by a professional photographer, that expresses his ideology.

\section{Aims and Objectives}

The aim of the current investigation was to identify the main reasons behind the perception of photogenic quality of public spaces in Colombo. Further it was attempted to explore the impact of social media on the above relationship under investigation. The criteria for this identification were set from the perspective of a spectacle user rather than a scholar with prior understanding of the given space. This situation analysis of so-called photogenic spaces is supposed to give insights to the delusions and reality of perception of the spectacle by the society. The results from the study can be used to create more meaningful 'symbols' in 
public spaces as the evolution of architectural manipulation comes to a crossroad with the growing social media universe.

\section{Theoretical Framework}

As elaborated by Sternberg \& Mio (1954) perception is of two-fold namely: constructive perception (topdown processing) and direct perception (ecological model) which laid the foundation for the study. Theoretical understanding on the visual perception of the spectator was based on the theories of Debord (1967).

\subsection{THE FACTORS OF VISUAL PERCEPTION}

The principal aspects of visual perception as clarified by Lauren \& Kayle.,( n.d.) are motivation, emotional state, past experiences, cultural background and context. These aspects can be further elaborated as below.

Motivation: Humans are driven by their motivations in perceiving the world. Motivation could be either, positive or negative, extrinsic and intrinsic, financial or non-financial. Intrinsic or internal motivation driven goals are goals that result in self-improvement. These goals are directly related to the process of the activity of perception. Extrinsic or exterior motivation driven goals are rather related to the outcomes of the activity of perception. They affect the wealth, status, etc which are long term motivators.

Emotional State: As stated by Goleman (1996) Emotional state is of fivefold, namely; self-awareness, selfregulation, motivation, social skills and empathy. Even though Goleman (1996) includes motivation as a part of emotional state it is taken as a separate element for the structuring of the framework for the study.

Cultural factor: Culture plays the most important part of an individual's worldview. Individual is structured from the birth to a thinking pattern adhering to the cultural norms. Cultural influence on individuality is higher in Asian countries than in European countries due to the strict rules of the society imposed upon the individualistic thinking. This psychological pressure shapes every action of the individual knowingly or unknowingly. Hofstede (1997) identifies seven elements of culture, explicitly social organization(money, job, education, heritage, caste, ethnicity, gender, age), customs/traditions, religion, government, language, arts/literature background and economic background.

Past Experiences: Past experiences are one of the strongest variables in affecting the decision-making process of an individual. But it cannot be sub divided since it's a rather personal factor changing from every user to user regardless of their other similarities.

Context: Context affects the way in which the object is perceived in a drastic way. Human mind is designed to identify objects in relative to the Contextual effects in the perception of area, length, orientation and lightness (Todorović, 2010).

\subsection{VISUAL ELEMENTS OF A PHOTOGRAPH}

Photograph itself is a combination of elements that are combined together in a two-dimensional plane to provide the viewer with a visually captivating image. The sense of beauty in this photograph is the culmination of these visual elements in harmony. An exact listing of these visual elements is quite hard to provide due to the subjectivity of these properties. Different photographers have provided different views on their ideology on the visual elements that compromises a photogenic image. Krist (2000), a leading photographer defines the prominent visual elements of a photograph as composition, lighting, colour balance and sense of moment. But contradicting to his idea Griffiths (2003) cited in Hall (2007) highlights on the direction, quality and temperature of light and how the lighting is focused on the subject as the most prominent character of a visually appealing photograph. It is further highlighted by Burian \& Caputo (2003) stating that the most beautiful photographs happen during the early mornings and sunsets. The colour temperature of warm lighting and also natural elements of fog, snow, frost and rain are identified by them as main catalysts in driving the ambience of a photograph to make them visually appeal (Hall, 2007).

But there are some common grounds that most of the photographers are agreed upon. That the photographs should have a subject and a background to highlight the subject. Without a subject a photograph would be monotonous. The context in which the subject is present defines the background of the photo. The addition of a foreground would highlight the subject more. So subject and context are main 
parts of visual appeal. Several factors have been identified by literature as visual elements in photography namely having a visible subject to the photograph, quality of light (; amount and temperature of ambient / artificial light to highlight the subject/ scenery), direction of light, light angle to highlight the subject of the photograph, sharpness or crispness of detailing that can be clearly identified in the situation, sense of moment, composition or arrangement of visual elements (unity, balance, movement, rhythm, focus, contrast, pattern, proportion), contrast/ brightness, colour balance and depth of the field.

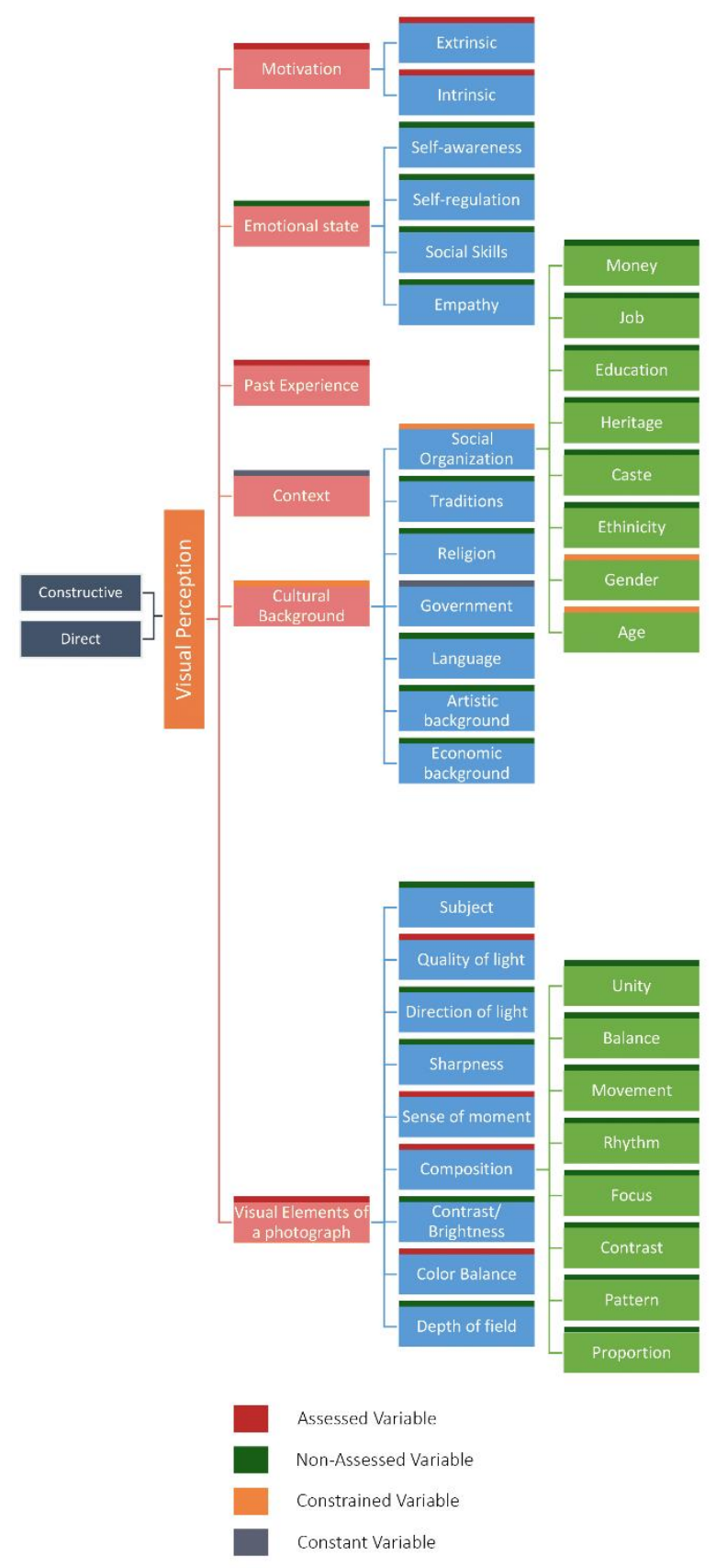

Figure 1 - Theoretical Framework for the study

Accordingly, considering the parameters of visual perception and visual elements the above illustrated theoretical framework was derived (figure 1). The variables which were assessed, constrained, not assessed and controlled in the study are marked with a corresponding colour as shown the legend.

\section{Methodology}

The methodology comprised of two stages; preliminary selection and online data gathering. 

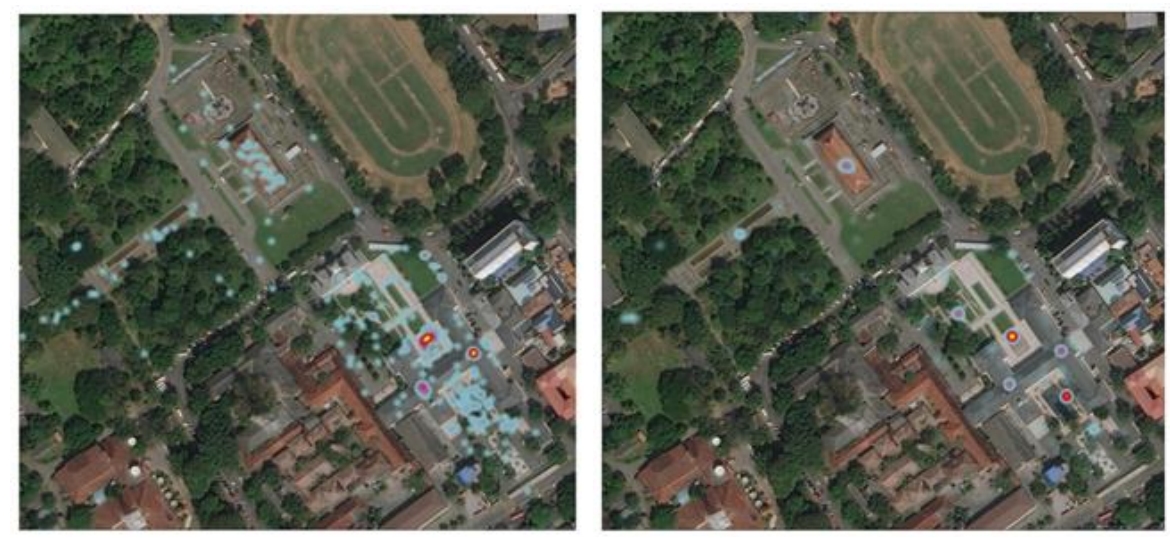

Figure 2 Scattered density of photogenic Figure 3 density of photogenic locations locations

The preliminary study was conducted via social media geo tagging hashtags of micro climax points within the identified public space. Three micro climax points were identified from online data analysis as most photogenic spaces within the premises. Independence Square in Colombo was taken as the generic public space for the preliminary study due to the diversity of user categories, diversity of spaces, historical value, larger area and the night functioning of the precinct.

A total of 300 online photos from Instagram were chosen randomly according to the "popular images" category and mapped as a density on a satellite map using ArcGIS Pro software. These points were converted into a density map to identify most photographed spaces. But the randomness of the points resulted in a scattered density as shown in Figure 2. As a means of clarifying clear points, random points were clustered depending on the zones they represent within the precinct. This resulted in giving out precise heat points, clearly showing the photographed spaces as shown in Figure 3.

Below 3 spaces were identified as the top 3 photographed spaces within the Arcade Independence Square premises, Colombo.

Space 1 - Location I - Area with the Lion Statues

Space 2 - Location E - Staircase area of the right-wing of the main building

Space 3 - Location F - Front area with the pond and fish tank facing the Bauddaloka Mawatha

Location I; area with the Lion statues was identified as the most popular image.

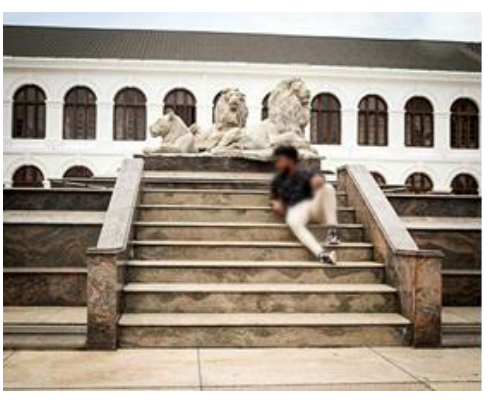

Figure 4 Space 1: Location I

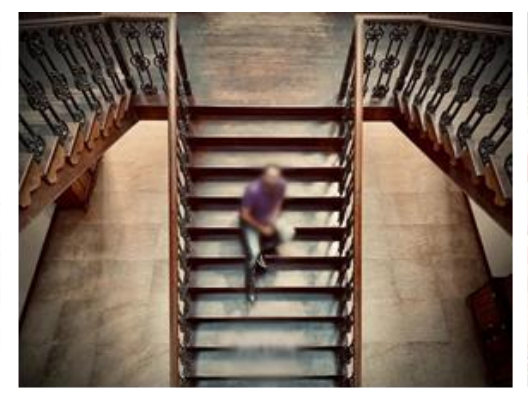

Figure 5 Space 2: Location E

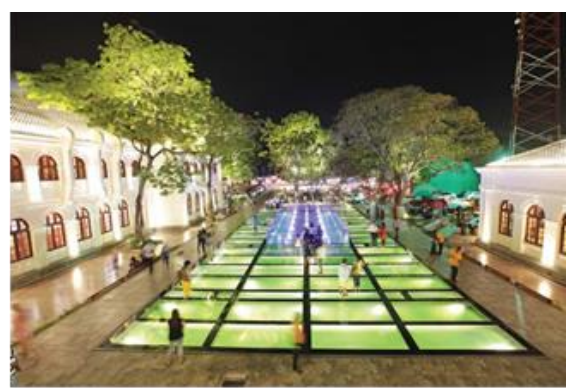

Figure 6 Space 3: Location F

\subsection{DATA GATHERING}

The photogenic quality of the three spaces selected in the preliminary study was studied at this level via questionnaires given to three user groups. This method of photographic analysis has been previously executed by Sugimotoa (2011) in his study of Hibiya Park in Japan and by Tok, Kaplan, \& Taneli (2010) in the study done on the city of Istanbul. Following three user groups were selected to study and the data gathering was done within a duration of 2 weeks. 
A - Professionals with architectural knowledge - 30 users

B - Professionals with photography knowledge -30 users

C - General public without professional knowledge on both architecture and photography - 30 users

An online questionnaire was prepared and the parameters from the theoretical framework was assessed adopting a 1-5 Likert scale, where rating of agreement increased from 1 to 5 . The questions were formulated to gather responses on social media usage, the perception of the photogenic nature of the selected spaces and factors influencing the perception of the photogenic nature. The following influencing factors were presented to the user in the questionnaire with the possibility of selecting the relevance of them using the Likert scale from 1 to 5 .

a. Extrinsic Motivation - Presenting it to others and outside world (including social media)

b. Intrinsic Motivation - Keeping as a personal trophy for self-satisfaction

c. Past Experience - Reminds of a past photo taken, past experience related to the specific place

d. Cultural factors - Influence of religion, art, traditions, history and socio-economic background

e. Visual Elements - Good quality of light available at the space, good composition, good colour balance and the sense of moment etc.

Emotional state was disregarded in the study as a parameter due to the online nature of the data collection. Each participant was given the photograph of the location as a reference in identifying the place prior to responding to the respective questionnaire, noting down whether they had seen the space earlier. Each group's responses about the level of photogenic quality of the location and responses to the variables was recorded.

\section{Research Findings}

\subsection{DATA ANALYSIS}

The responses were categorised under the 3 user categories and the data sheets were analysed in Microsoft Excel.

A - Professionals with architectural knowledge - 33 responses

B - Professionals with photography knowledge - 31 responses

C- General public without professional knowledge on both architecture and photography - 30 responses

Analysing the data, it was evident that more than $75 \%$ of every user group spent more than 1 hour on social media on a daily basis, and the majority spends more than 2 hours online. The influence of media on the decision-making process increases with time spent on social media. The average spectacle user does not identify the impact of influences given by the media, in making the user belong to a commodity of the government and mass media itself (Debord, 1967).

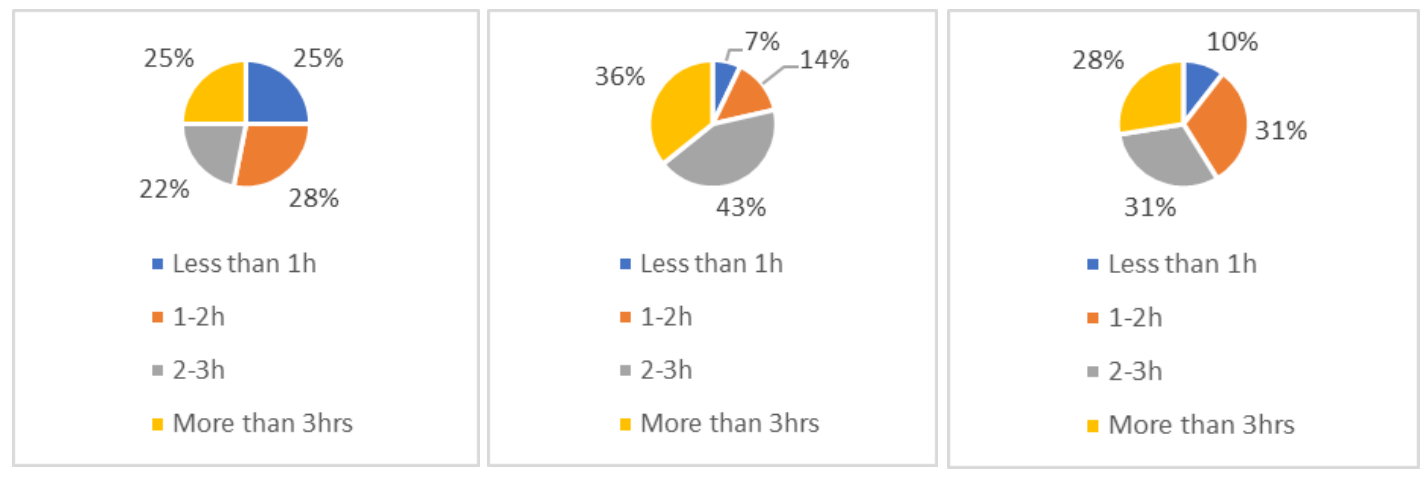

Figure 7 Social media usage of Group A
Figure 8 Social media usage of Group B
Figure 9 Social media usage of Group C 


\subsubsection{Space 1 - Location I}

The level of photogenic quality of space 1 was quantified as per the below graphs. The results between the groups were quite contrasting to each other. Group A with architects had mixed feelings about the level of photogenic quality. Group B with more photographic expertise had expressed the photogenic quality more towards being neutral. Quite contrastingly group $\mathrm{C}$ viewed the photo as being more photogenic.

The reasoning behind the level of photogenic quality was analysed next. Visual elements of the composition and the extrinsic motivation had been the main driving factors while the past experience had the lowest tendency. Groups A and B had opted that this place is less of a photogenic space while Group C had given more positive feelings of photogenic quality in this space. But the Likert graphs did not provide with a clear difference between the opinions of groups.

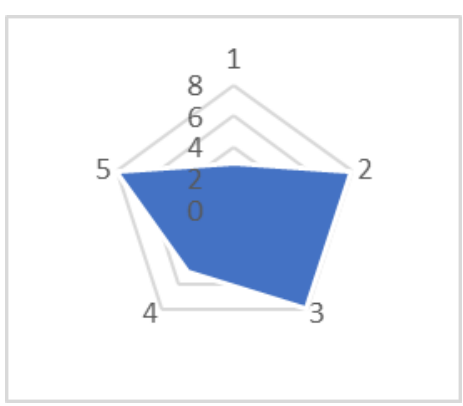

Figure 10 Level of photogenic quality of space 1 by Group A

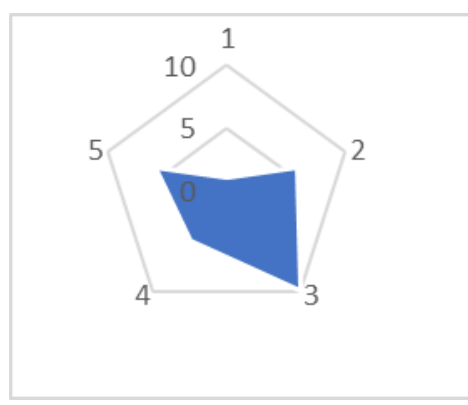

Figure 11 Level of photogenic quality of space 1 by Group B

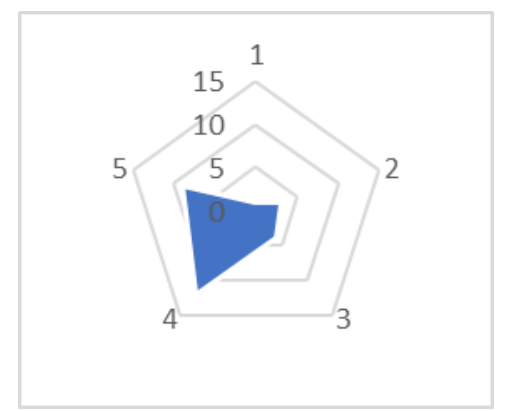

Figure 12 Level of photogenic quality of space 1 by Group C

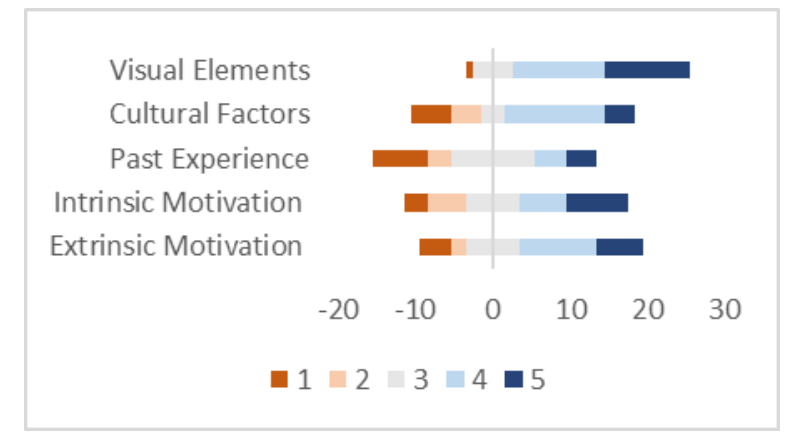

Figure 13 Likert analysis of Space 1 photogenic quality by group A

Visual Elements

Cultural Factors

Past Experience Intrinsic Motivation Extrinsic Motivation

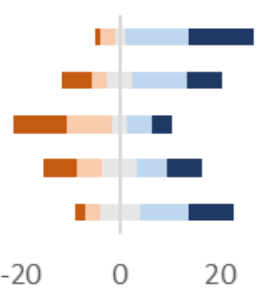

40

$\square 1 \square 2 \square 3-4 \square 5$

Figure 15 Likert analysis of Space 1 photogenic quality by group $\mathrm{C}$

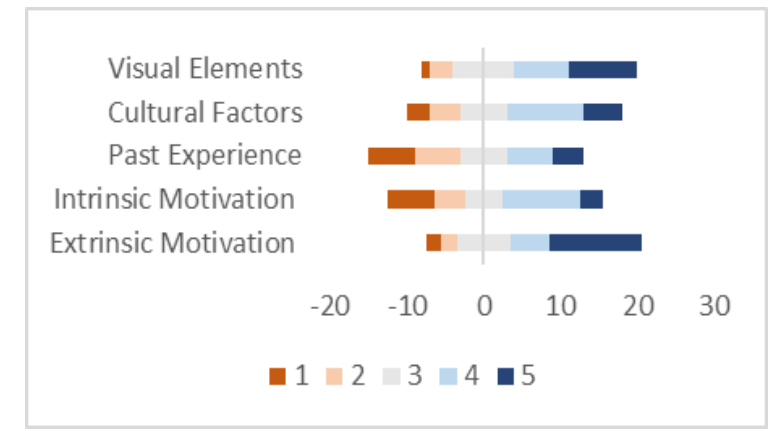

Figure 14 Likert analysis of Space 1 photogenic quality by group B

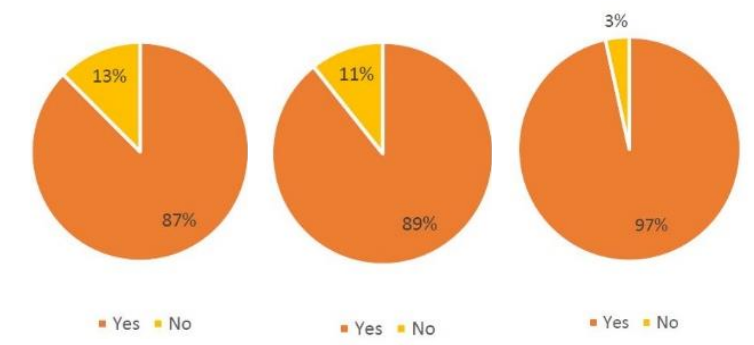

Figure 16 Previous Sightings of Photo of space 1 by Group A, B and C respectively

$59 \%$ of group C have spent more than 2 hours daily on social media (Fig 9). But evidence was not quite clear to reason out a connection between the time spent in social media with the results obtained from quantifying photogenic quality. But the results from analysis clearly showed that (figure 16) a majority $(97 \%)$ have seen this photo previously from social media while the figure for groups A \& B stood at $87 \%$ and $89 \%$ respectively. This data builds up a connection between the influence of social media and the perception of the photogenic quality of space 1 . 


\subsubsection{Space 2 - Location E}

The results between the groups were not as contrasting as Space 1. All the groups had a favourable positive reaction depicting the space being more of a photogenic space. The reasoning behind the level of photogenic quality did not show a clear perception pattern. The visual elements were considered as the main quantified influence by every group. The general deviation of perception factors showed the same trend in both groups $\mathrm{A}$ and $\mathrm{B}$. This had been changed in group $\mathrm{C}$ where a deviation was shown from the intrinsic motivation factor. For group $\mathrm{C}$ intrinsic motivation had been perceived more than extrinsic motivation.

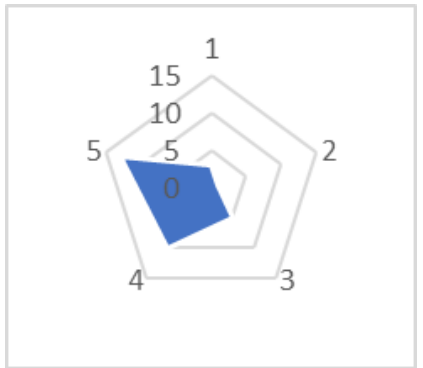

Figure 17 Level of photogenic quality of space 2 by Group A

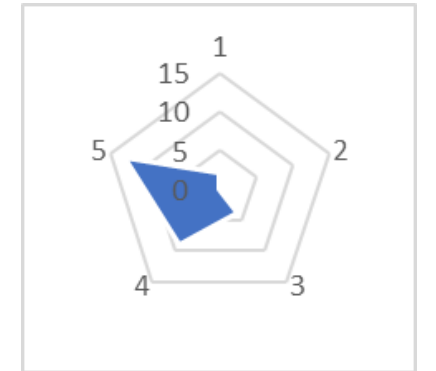

Figure 18 Level of photogenic quality of space 2 by Group B

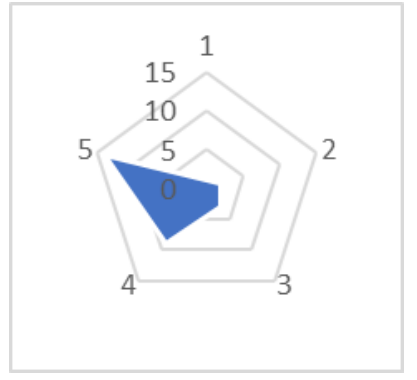

Figure 19 Level of photogenic quality of space 2 by Group C

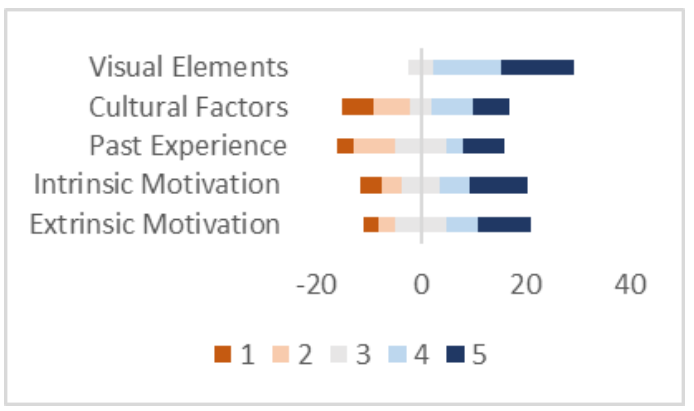

Figure 20 Likert analysis of Space 2 photogenic quality by group A

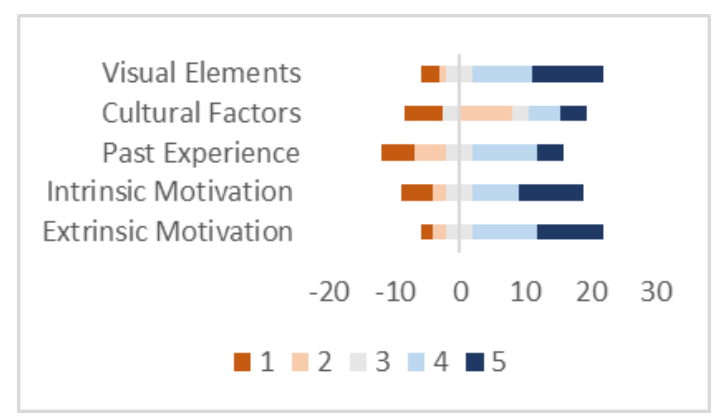

Figure 22 Likert analysis of Space 2 photogenic quality by group $\mathrm{C}$

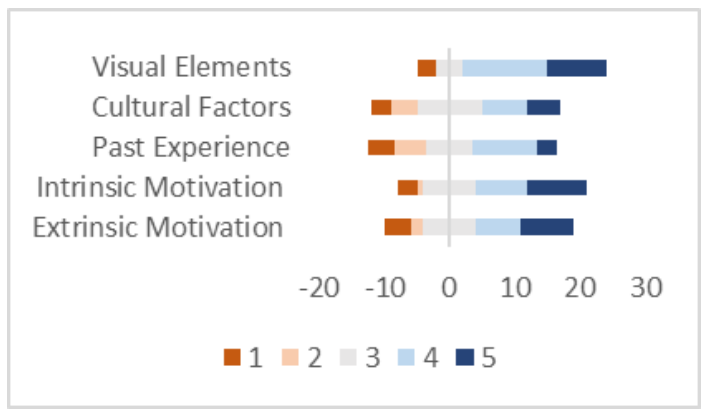

Figure 21 Likert analysis of Space 2 photogenic quality by group B

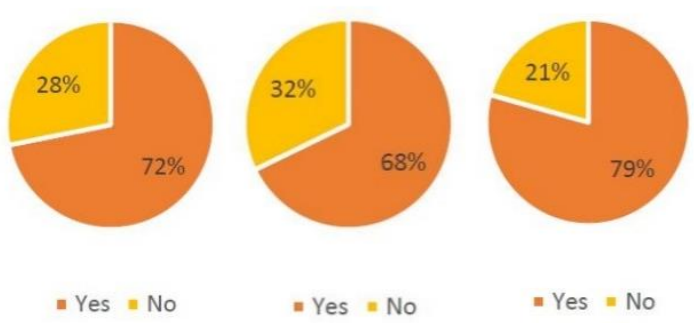

Figure 23 Previous Sightings of Photo of space 2 by Group A, B and C respectively

But this alone was not able to build up any relationship with the previous data. A further study should be done to explore why group $\mathrm{C}$ had more intrinsic motivation than extrinsic towards perceiving the photogenic quality of the space. But this trend does not relate with the other 2 spaces and could be taken as a random event considering the number of samples in the data set being rather low.

\subsubsection{Space 3 - Location F}

The level of photogenic quality between the groups was found to be contrasting with each other with reference to space 3 . While groups $A$ and $C$ had a more positive quantified reaction, group B had mixed 
positive notions. But groups A and C predominantly displayed the reaction to be a very much positive quantification.

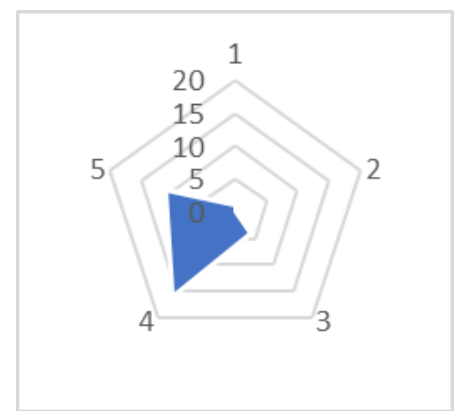

Figure 24 Level of photogenic quality of space 3 by Group A

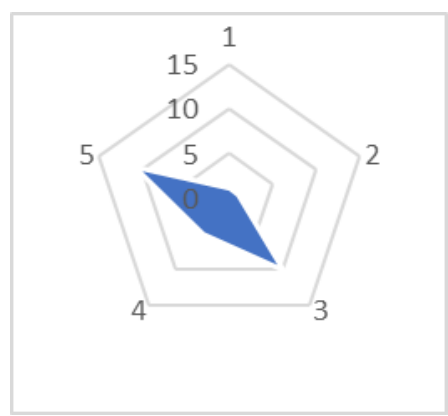

Figure 25 Level of photogenic quality of space 3 by Group B

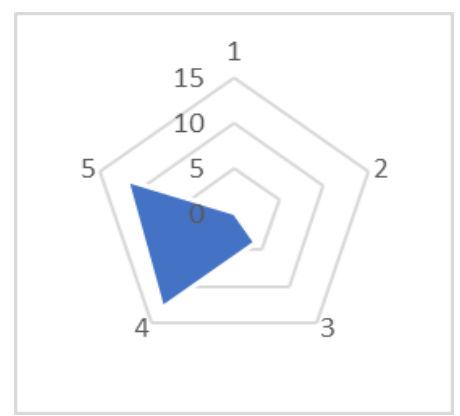

Figure 26 Level of photogenic quality of space 3 by Group C

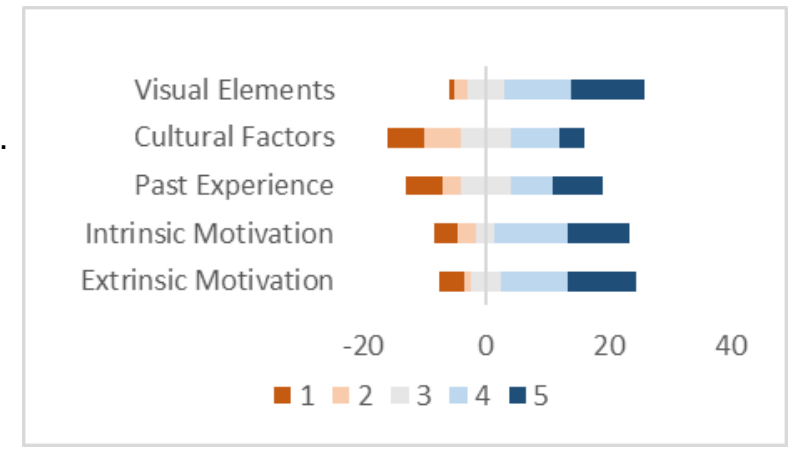

Figure 27 Likert analysis of Space 3 photogenic quality by group $\mathrm{A}$

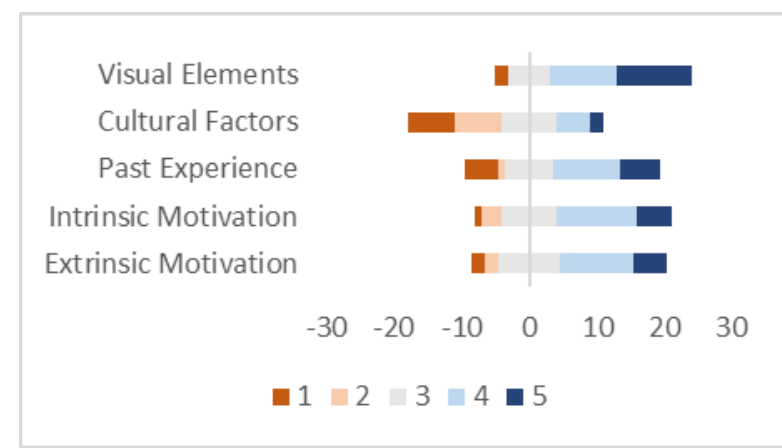

Figure 29 Likert analysis of Space 3 photogenic quality by group $\mathrm{C}$

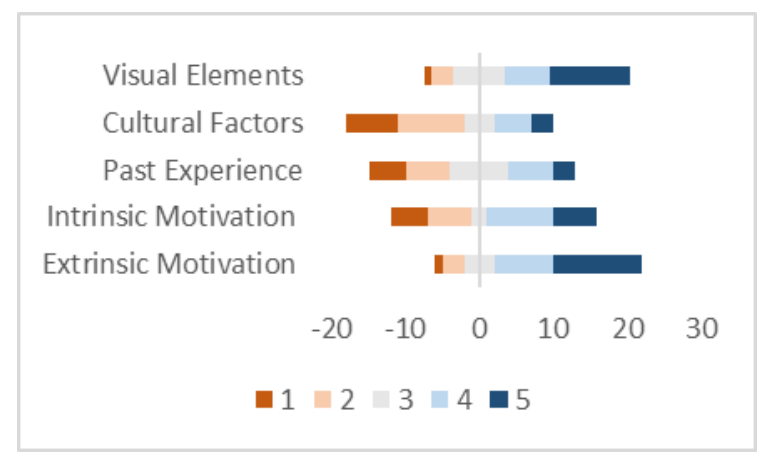

Figure 28 Likert analysis of Space 3 photogenic quality by group B
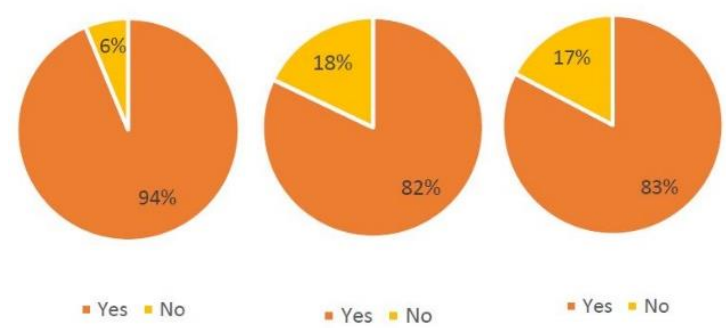

Figure 30 Previous Sightings of Photo of space 3 by Group A, B and C respectively

The Likert analysis did show a clear trend between group A and B while group C shared a different deviation pattern. All the groups have rated visual elements as the highest influential factor towards perception with reference to space 3. Influence of culture had been rated the lowest. The major difference between the trends was the influence of extrinsic motivation. Just like in the space 2 Likert analysis of group $\mathrm{C}$ did provide a similar deviation showing less influence from extrinsic motivation, while intrinsic motivation emphasized more impact on the perception.

The group A had higher influence from media on the perception of space 3 . They had seen this space before from social media than group B \& C. And group A had extrinsic motivation towards publishing this image back into social media sites. This indicated a connection between the spatial perception being influenced by media. But Group B had the highest extrinsic motivation than any other group. Further studies should be done to see why Group B had mixed feelings while they had extrinsic motivation. In general, spaces 1 and 3 showed clear influence of media on the perception of spaces as photogenic. The impact was not limited to a certain group type whereas group $\mathrm{C}$ was influenced in space 1 while the groups 
$\mathrm{B}$ and $\mathrm{C}$ got influenced in space 3 . Space $2 \mathrm{had}$ a complicated pattern that needed further data to come to a definite conclusion. But the clear trend of deviation of group $C$ in space 2 and 3 should be observed in future studies due to the evident pattern from this study.

\section{Conclusion}

Visual elements of the frame or the space was identified as the main reason behind the perception of photogenic quality of a space across all the three spaces tested. This was common to all the user groups studied. Cultural influence was identified as the lowest influential factor towards visual perception of photogenic spaces. The understanding of the motivational factors generated unexpected findings where group C (general public) who did not have any photography or architecture related expertise, considered the intrinsic motivation to be more of an influential factor than the extrinsic motivation. On one hand this shows that professionals use photos more as a means of reaching out to the public, understanding the current needs of the public and to get feedback. Also, on the other hand it is noteworthy that the selfactualization is a subconscious process. But since the influence of media on self-actualization is on a subconscious level the user may not necessarily realize the influence on media on their cognitive decisionmaking process. Therefore, even though the user thinks the motivation as more intrinsic in fact it has underlying extrinsic motivational factors without the users' realization. This is further discussed by Guy Debord in his thesis in detail (Debord, 1967).

A connection was identified among the level of photogenic quality and the influence from social media. In space 1 - Group C had seen the space before from social media the most. And Group C had the highest percentage of users thinking that space 1 was very photogenic. Similarly, in space 3 - Group A had seen this space more than other groups and they had more extrinsic motivation towards perception of visual photogenic quality contributing the photographs back to social media feeds. Therefore, a complete cycle is created from seeing to resubmitting of spaces in 2D photos which at the same time influences the visual perception.

\subsection{RECOMMENDATIONS FOR FUTURE STUDIES}

It is noteworthy that even though in the preliminary study space A; lion statue area, was identified as the most popular area to be photographed, which was derived via scrutinizing 300 photos from the popular social media feeds, none of the user groups in the online data gathering recommended it as being photogenic. Therefore, there is a contradiction between the public social media opinion and the studied user data. The reasoning behind this is not clear. This has to be further examined in-depth in future with a higher number of sample photos taken from social media or with more participation from Group C user category. Also, further studies could be conducted into specifically quantifying the extrinsic motivation and intrinsic motivation of user groups towards spatial perception. And this could be further developed into finding the connection between the influence of other factors on the motivational aspects of visual perception.

\section{References}

Burian, P. \& Caputo, R. (2003). National geographic photography field guide. Washington, D.C.: National Geographic. Debord, G. 1967, The society of the spectacle. France: Buchet-Chastel.

Godfrey, A., \& Gretzel, U. 2010, The Use of Modern Architecture in City Marketing. Travel and Tourism Research Association: Advancing Tourism Research Globally. 33.

Goleman, D. (1996). Emotional Intelligence. Why It Can Matter More than IQ. Learning, 24(6), pp. 49-50.

Hall, J. L. (2007). Elements that influence visual appeal in photographs. Ohio: Graduate College of Bowling Green.

Hofstede, G. (1997). Cultures and Organizations: Software of the mind. New York: McGraw Hill.

Humphreys, L. 2010, Mobile social networks and urban public space. New Media \& Society XX(X), 1-16.

Kima, H., Chae, B., \& Park, S. 2017, Exploring public space through social media:an exploratory case study on the High Line New York City. Urban Design International 23,2, 69-85.

Krist, B. (2000). Spirit of place. New York, NY: Amphoto Books.

Lauren \& Kayle. (n.d.). Perceptual set - Influences on our interpretation of visual information. Retrieved from Visual Perception: https://visual-perception.weebly.com/perceptual-set.html

Sugimotoa, K. 2011, Analysis of Scenic Perception and Its Spatial Tendency: Using Digital Cameras, GPS loggers, and GIS. Procedia Social and Behavioral Sciences 21, 43-52.

Sternberg, R., \& Mio, J. (1954). Cognitive Psychology (Fourth Edition ed.). Wadsworth, London: Thomson Learning. Todorović, D. (2010). Context effects in visual perception. Review of Psychology, Vol.17, No.1, pp. 17-32. 
Tok, S. Y., Kaplan, I., \& Taneli, Y. 2010, Photography in architectural education: A tool for assessing social aspects of the built environment. Procedia Social and Behavioural Sciences 2, 2583-2588. 